\title{
A new kinetic model for the accumulation of poly(3-hydroxybutyrate) in Alcaligenes eutrophus, 1
}

\section{Granule growth}

\author{
Jenci Kurja*, Hennie F. Zirkzee \\ Department of Polymer Chemistry and Technology, Eindhoven University of \\ Technology, P. O. Box 513, 5600 MB Eindhoven, The Netherlands
}

\section{Gertjan M. de Koning}

Institute of Biotechnology, ETH-Hönggerberg HPT D75, CH 8093 Zürich, Switzerland

\section{Ian A. Maxwell}

Memtec, 1 Memtec Parkway, South Windsor, NSW 2756 Australia

(Received: January 31, 1995)

\section{SUMMARY:}

A new kinetic model is proposed for the accumulation of poly(3-hydroxybutyrate) (PHB) in Alcaligenes eutrophus. The model developed is closely related to the descriptive model of Harkins for the emulsion polymerization process. The PHB accumulation is discussed in terms of polymerization events such as initiation, propagation and termination, taking into account the heterogeneous nature of the system. Experimental results on the PHB accumulation in Alcaligenes eutrophus can be described in both a quantitative and qualitative manner by the model. Further, it is shown that the decrease in the rate of polymerization during the PHB accumulation can be explained by coalescence of the granules. The decrease of the molecular weight of the polymer formed during the accumulation process can be fully explained in terms of the kinetic mechanisms of progapation and chain transfer, and the effect of granule size on these.

\section{Introduction}

Since Lemoigne" first isolated and characterized the poly $((R)$-3-hydroxybutyrate) (PHB) homopolymer, PHB has been extensively studied by biochemists to elucidate its biosynthetic pathways ${ }^{2,3)}$. One year later a patent was granted to Dinsmore ${ }^{4)}$ working for the Goodyear Tire \& Rubber Company for a process which can be regarded as the first true emulsion polymerization. It describes the thermal polymerization over a period of six months of aqueous emulsions of various diene-monomers, with oleate salts and egg albumin as stabilizers.

It was not until 1968 that Ellar et al. ${ }^{5)}$, who reported on the morphology of poly $(\beta$-hydroxybutyrate) granules, drew some equivalents with the emulsion polymeri- 
zation process as quoted: "The model that has been developed is not too unlike the familiar emulsion polymerization equation, except that the number of chain initiation sites in this case is probably determined by a well-defined number of protein molecules which form the initial micelle".

Ballard et al. ${ }^{6}$ have drawn the conclusion that the PHB biosynthesis has some of the features of an emulsion polymerization with the individual granules corresponding to latex particles and the membrane lipid to surfactant. Though, they did not consider PHB biosynthesis as an emulsion polymerization since the PHB polymerization mechanism is not free radical but a coordination-insertion process bearing more resemblance to a Ziegler-Natta type system.

Very recently, Gerngross et al. ${ }^{7)}$ proposed a model for granule formation. They assume that granule formation is caused by the formation of micelles formed from polymerase enzyme molecules which are propagating in the cytoplasm.

At the same time, De Koning and Maxwel1 ${ }^{8)}$ drew an analogy between the conventional emulsion polymerization process and the biosynthesis of poly(3-hydroxyalkanoate)s. They stated that the granule formation mechanism will closely resemble a homogeneous nucleation mechanism, i.e. polymerase enzyme molecules which propagate in the cytoplasm form precursor granules. These precursor granules then grow until they are mature granules.

Here, we present a more extensive study in which we extend and quantify the ideas proposed by De Koning and Maxwell ${ }^{8)}$ for the granule growth stage of the PHB accumulation process. The quantification of the granule formation stage will be discussed elsewhere ${ }^{9)}$.

Before we discuss the analogues between the bacterial PHB accumulation and a conventional emulsion polymerization we would like to give a short overview of these processes as they are currently accepted in the open literature.

\section{Emulsion polymerization}

Emulsion polymerization is often referred to as a particular case of free radical polymerization, although other types of polymerization reactions in emulsion are known, e.g. the emulsion polymerization of siloxanes ${ }^{10-12)}$ and the ring-opening metathesis polymerization of oxanorbornene derivates ${ }^{13}$. The kinetics and mechanisms involved in an emulsion polymerization are highly complicated, since events occur in several phases, and exchange of radicals and monomer between these phases need to be taken into account. Models and theories dealing with these aspects of emulsion polymerization are numerous and excellent reviews are available ${ }^{14-16)}$.

In contrast to bulk and solution polymerization, emulsion polymerizations are heterogeneous reaction systems ${ }^{17}$. Generally an emulsion polymerization system comprises of water, monomer, surfactant, initiator and additives such as chain transfer agents and buffers. A widely used and accepted mathematical model for the emulsion polymerization process is that of Smith and Ewart ${ }^{18)}$, which is based on the descriptive model of Harkins ${ }^{19}$. The Harkins model subdivides the emulsion polymerization process into three distinct intervals. A typical conversion-time curve is shown in Fig. 1. For simplicity we will assume that the added surfactant is present at 
Fig. 1. A typical conversion-time curve for an emulsion polymerization with the different intervals indicated

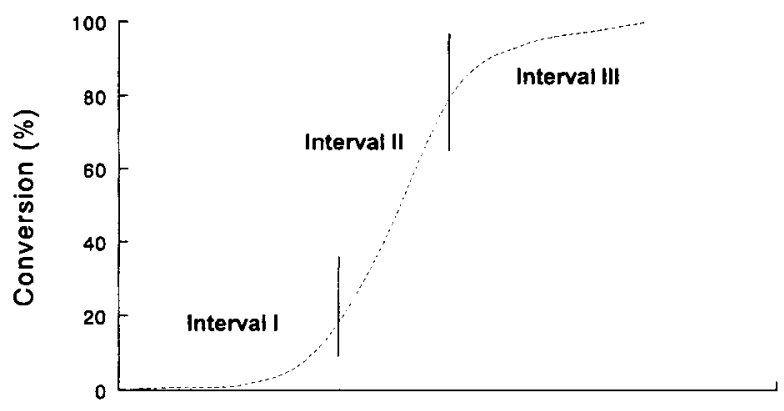

Time

a concentration above the $\mathrm{cmc}$ (=critical micelle concentration), although this is not necessary since there are several examples of emulsifier free emulsion polymerizations described in the literature (cf., e.g., ref. ${ }^{20)}$ ).

Interval $I$ is the initial stage where particle formation takes place via several possible mechanisms ${ }^{14,17,21,22)}$. The initiator (oil or water soluble) dissociates into reactive radicals. These radicals will mainly diffuse to the monomer swollen micelles, since their total surface is much bigger than that of the monomer droplets. In the monomer swollen micelles the radicals will initiate the polymerization. This process is also referred to as micellar nucleation ${ }^{(8,19)}$. There is also evidence that before the radical enters the micelle it has gained a certain length by addition of monomer present in the water phase ${ }^{14}$.

Interval II is entered when no new particles are being formed and is characterized by the fact that the number of polymer particles reaches a constant value. Polymerization in the particles proceeds in the presence of a separate monomer phase. The monomer droplets ensure a constant transport of monomer to the growing polymer particles (no diffusion limitation), i.e. the rate of diffusion of monomer from the monomer droplets to the water phase and subsequently to the polymer particles is higher than the rate of consumption of the monomer in the polymerization process. The monomer and radical concentration within the water phase and the polymer particles is constant during interval II, leading to a constant rate of polymerization.

Interval III begins with the disappearance of the monomer droplets, after which the monomer concentration in the polymer particles decreases continuously. Concomitantly the rate of polymerization decreases. For clarity we would like to emphasize that the polymerization takes place inside the growing polymer particles, although exceptions to this are known such as the emulsion polymerization of acrylonitrile and tetrafluoroethylene (cf., e.g., ref. ${ }^{23)}$ ).

In general, the rate of a free radical polymerization $\left(R_{\mathrm{p}}\right)$ is given by:

$$
R_{\mathrm{p}}=k_{\mathrm{p}} \cdot(\mathrm{M}) \cdot(\mathrm{R})
$$


where $k_{\mathrm{p}}$ is the second-order propagation rate coefficient, (M) and (R) the monomer and free radical concentration, respectively. In emulsion polymerization the overall rate of polymerization can be taken as the summation of those in each individual polymer particle, leading to

$$
R_{\mathrm{p}}=k_{\mathrm{p}} \cdot C_{\mathrm{M}} \cdot \bar{n} \cdot N / N_{\mathrm{Av}}
$$

where $C_{\mathrm{M}}$ is the monomer concentration within the polymer particles, $\bar{n}$ the average number of radicals per particle, $N$ the number of polymer particles per unit volume, and $N_{\mathrm{Av}}$ Avogadro's number.

\section{Accumulation of poly(3-hydroxybutyrate)}

\section{Metabolism of PHB synthesis}

Since PHB polymer is a storage material, it is appropriate to consider its metabolism as a cycle of synthesis and breakdown ${ }^{2,3)}$. The enzymes of the cycle have been examined extensively in a range of organisms and found to be very similar. The carbon source supplied to the bacterium is metabolized, via several steps, into $(R)$-3-hydroxybutyryl-CoA (CoA: coenzyme A). This compound is believed to be the monomer that is converted by the polymerase enzyme into $\mathrm{PHB}$.

\section{Kinetic models for $\mathrm{PHB}$ biosynthesis}

The kinetics of the synthesis of biopolymers was recently reviewed by Luong et al. ${ }^{24)}$ The models discussed ${ }^{25-27)}$ involve mainly the growth kinetics of the bacteria while the polymer accumulation process was not examined in any great detail. Kawaguchi and $\mathrm{Doi}^{28)}$, on the other hand, proposed a reaction equation for the polymerization process of PHB, based on the usual initiation, propagation and termination concept used in polymer chemistry. However, they didn't take into account the heterogeneous character of the PHB polymerization system.

\section{Physical model for the PHB accumulation in Alcaligenes eutrophus}

When considering any polymerization process, the first and simplest approach is to consider a homogeneous system, i.e. the concentration of all reactants is equal throughout the reaction system. However, this can not be used in the present case because the PHB polymerization system is inherently heterogeneous.

\section{Formation of granules}

Only initially the system is homogeneous. The polymerase enzyme is present in the cytoplasm $^{7,29,30)}$, and there are no granules present. Once the bacterium starts accumulating PHB, monomer will react with the polymerase enzyme in the cytoplasm, hence initiation has occurred. During the propagation the polymerase enzyme will stay bound to the growing polymer chain. The polymerization leads to the formation of 
oligomeric polymerase enzyme molecules. The oligomeric polymerase enzyme molecule can be regarded as a surface active species, where the polymerase enzyme is the hydrophilic headgroup while the polymer chain is the hydrophobic tail.

At a certain point the growing chain will precipitate since it exceeds its water solubility, or in other words, a colloidal particle will be formed in order to minimize its hydrophobic surface area. The so-formed hydrophobic surface will attract phospholipids and other surface active cell constituents. The granule surface can also be stabilized by the endgroups of polymer chains present, i. e. the hydroxyl or carboxyl endgroups can act as cosurfactant. This stage of the PHB accumulation is similar to interval $\mathrm{I}$ in the conventional emulsion polymerization process. A more quantitative and extensive approach of granule formation will be discussed in detail elsewhere ${ }^{9}$.

\section{Growth of granules}

Once the stage of granule formation is completed, the bacteria enter the stage of granule growth. This stage of PHB accumulation is characterized by two main features: first, the constant number of granules per bacterium, being typically $8-14$ granules per bacterium for Alcaligenes eutrophus ${ }^{6}$; secondly, a constant rate of polymerization. The granule growth stage can be compared to interval II of a conventional emulsion polymerization process, as the number of polymer particles, i.e. loci of polymerizations, remains constant ${ }^{14)}$. Monomer diffuses to the surface of the granule where it is converted into polymer by the granule-associated polymerase enzyme ${ }^{7}$. If a growing polymerase enzyme molecule has undergone chain transfer it will desorb from the granule surface due to its hydrophilic character. In the cytophasm, a free polymerase enzyme will grow into an oligomeric polymerase enzyme and precipitate onto the granule surface rather than forming a new granule as in interval 1 .

The propagation in the case of PHB accumulation occurs mainly at the surface of the granules which is somewhat different from the conventional emulsion polymerization process where the propagation of the polymer chain occurs inside the latex particles. Another difference lays in the monomer supply. The presence of a separate monomer phase in a conventional emulsion polymerization ensures a constant monomer concentration in the polymer particles. In the case of the PHB accumulation monomer is continuously supplied by a cascade of reactions in the cytoplasm.

It is known that the bacteria can continue accumulating PHB up to a weight fraction PHB of approximately 0.75 , at which point PHB accumulation stops, although there is still monomer and active polymerase enzyme present ${ }^{6)}$.

\section{Kinetic model for the accumulation of PHB in Alcaligenes eutrophus}

In previous sections we have formulated the different stages of PHB accumulation in terms of the emulsion polymerization process. We will now put these results together into a kinetic model for PHB accumulation, or more specifically the granule growth stage. At this point it is not our aim to develop a detailed kinetic model, rather a framework from which further improvements can be incorporated. 
Initiation

A polymerase enzyme molecule has to be initiated in the cytoplasm. The overall initiation reaction is given by Eq. (3).

$$
\mathrm{E}-\mathrm{SH}+\mathrm{M}-\mathrm{SCOA} \stackrel{k_{\mathrm{i}}}{\longrightarrow} \mathrm{M}-\mathrm{S}-\mathrm{E}+\mathrm{CoA}-\mathrm{SH}
$$

Here E-SH denotes the free polymerase enzyme, M-SCoA the monomer, CoA-SH is coenzyme-A and M-S-E is a polymerase enzyme with one monomer unit attached to it. $k_{\mathrm{i}}$ is the second-order initiation rate coefficient. The rate of initiation $\left(R_{\mathrm{i}}\right)$ is given by Eq. (4):

$$
R_{\mathrm{i}}=k_{\mathrm{i}} \cdot(\mathrm{E}-\mathrm{SH}) \cdot(\mathrm{M}-\mathrm{SCOA})
$$

Here (M-SCoA) denotes the monomer concentration in the cytoplasm and (E-SH) the concentration of free polymerase enzyme in the cytoplasm.

\section{Propagation in the cytoplasm}

Once the polymerase enzyme molecule has been initiated, it can propagate in the cytoplasm according to Eq. (5):

$$
\mathrm{M}_{n}-\mathrm{S}-\mathrm{E}+\mathrm{M}-\mathrm{SCOA} \stackrel{k_{\mathrm{p}}^{\mathrm{c}}}{\longrightarrow} \mathrm{M}_{n+1}-\mathrm{S}-\mathrm{E}+\mathrm{CoA}-\mathrm{SH}
$$

Here $\mathrm{M}_{i}-\mathrm{S}-\mathrm{E}$ represents the polymerase enzyme in the cytoplasm which is attached to a polymer chain with a degree of polymerization of $i(=n \& n+1)$, and $k_{\mathrm{p}}^{\mathrm{c}}$ is the second-order propagation rate coefficient for propagation in the cytoplasm.

Adsorption of oligomeric polymerase enzyme molecules onto granules

At a degree of polymerization of $z$ the polymerase enzyme molecule which propagates in the cytoplasm will become surface active and can adsorb onto a granule. Adsorption of these oligomeric polymerase enzyme molecules onto granules can be envisaged by Eq. (6):

$$
\mathrm{M}_{z}-\mathrm{S}-\mathrm{E}+\text { Total Granule Surface } \stackrel{k_{\mathrm{ads}}}{\longrightarrow} \text { Adsorption }
$$

Here $k_{\text {ads }}$ is the adsorption rate coefficient and $\mathrm{M}_{z}-\mathrm{S}$-E the adsorbing species with a degree of polymerization of $z$. It is not too unlikely to assume that species with a degree of polymerization of $z \pm s$ also give adsorption. Here, $s$ is an integer which is a measure for the distribution of adsorbing oligomeric polymerase enzyme molecules. However, for simplicity we will only take the average degree of polymerization of $z$ into account. Adsorption implied by Eq. (6) is supposed to be irreversible. The rate of adsorption $R_{\mathrm{a}}$ (in $\mathrm{mol} \cdot \mathrm{L}^{-1} \cdot \mathrm{h}^{-1}$ ) can be easily visualized by Eq. (7):

$$
R_{\mathrm{a}}=k_{\text {ads }} \cdot\left(\mathrm{E}-\mathrm{S}-\mathrm{M}_{z}\right) \cdot A_{\text {gran }}^{\mathrm{t}}
$$


Here, $A_{\text {gran }}^{\mathrm{t}}$ is the total granule surface area per litre of reaction mixture, which is equal to

$$
A_{\mathrm{gran}}^{\mathrm{t}}=\pi \cdot d_{\mathrm{gran}}^{2} \cdot N_{\mathrm{gran}}
$$

where $d_{\text {gran }}$ denotes the average granule diameter and $N_{\text {gran }}$ the number of granules per litre of reaction mixture.

Propagation at the granule surface

After adsorption has occurred the polymerase enzyme molecule can propagate at the granule surface. The propagation step at the granule surface can be visualized by Eq. (9).

$$
\mathrm{M}_{i}-\mathrm{S}-\mathrm{E}+\mathrm{M}-\mathrm{SCOA} \stackrel{k_{\mathrm{p}}}{\longrightarrow} \mathrm{M}_{i+1}{ }^{-} \mathrm{S}-\mathrm{E}+\mathrm{CoA}-\mathrm{SH}
$$

We assume that the rate of propagation is independent of the length of the polymer chain attached to the polymerase enzyme (Flory's principle of equal reactivity) ${ }^{31)}$. The rate of PHB accumulation or the rate of polymerization, $R_{\mathrm{p}}$ (in $\mathrm{mol} \cdot \mathrm{L}^{-1} \cdot \mathrm{h}^{-1}$ ), is given by

$$
R_{\mathrm{p}}=k_{\mathrm{p}} \cdot(\mathrm{M}-\mathrm{SCOA})^{\mathrm{Gran}} \cdot\left(\mathrm{E}-\mathrm{S}-\mathrm{M}_{i}\right)
$$

where (M-SCOA $)^{\mathrm{Gran}}$ is the monomer concentration at the granule surface which is not necessarily equal to the monomer concentration in the cytoplasm. The monomer (see Fig. 2) bears some resemblance with biosurfactants such as rhamnolipids ${ }^{32}$.

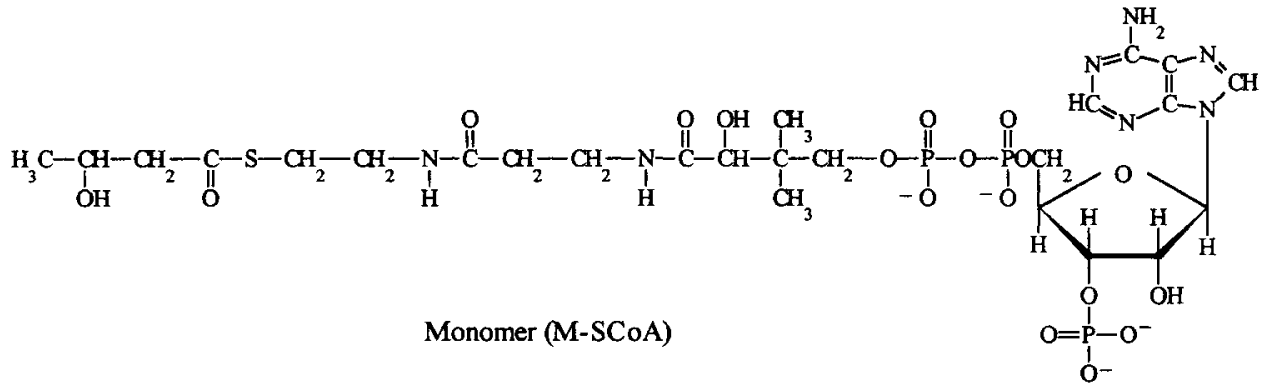

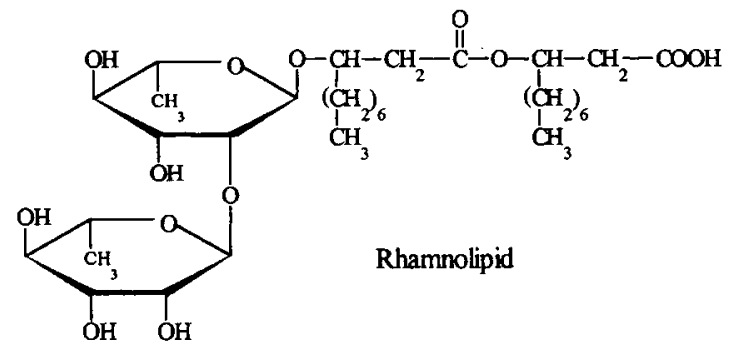

Fig. 2. Structural formula of the monomer (M-SCoA) and a rhamnolipid 
Therefore, it is assumed that the monomer exhibits surface active properties. (E-S-M $\left.\mathbf{M}_{i}\right)$ is the concentration of the propagating species at the granule surface with a degree of polymerization $i$, while $i>z$, while $k_{\mathrm{p}}$ is the second-order propagation rate coefficient for propagation at the granule surface. Assuming that most of the polymerization takes place at the granule surface, the overall rate of polymerization $R_{\mathrm{p}}$ can be taken as the summation over the individual granules. This leads to Eq. (11) which is comparable to Eq. (2) for the emulsion polymerization process

$$
R_{\mathrm{p}}=\left(k_{\mathrm{p}} \cdot(\mathrm{M}-\mathrm{SCOA})^{\mathrm{Gran}} \cdot n^{*} \cdot N_{\mathrm{gran}}\right) / N_{\mathrm{av}}
$$

where $n^{*}$ is the average number of active polymerase enzyme molecules per granule.

Chain transfer at the granule surface

We assume that chain transfer to monomer and/or water can occur as depicted by Eqs. (12) and (13), respectively.

$$
\begin{aligned}
& \mathrm{E}-\mathrm{S}-\mathrm{M}_{n}+\mathrm{M}-\mathrm{SCoA} \stackrel{k_{c t}^{\mathrm{M}}}{\longrightarrow} \mathrm{E}-\mathrm{S}-\mathrm{M}+\mathrm{M}_{n}+\mathrm{CoA}-\mathrm{SH} \\
& \mathrm{E}-\mathrm{S}-\mathrm{M}_{n}+\mathrm{H}_{2} \mathrm{O} \stackrel{k_{\mathrm{ct}}^{\mathrm{w}}}{\longrightarrow} \mathrm{E}-\mathrm{SH}+\mathrm{M}_{n}
\end{aligned}
$$

Here $k_{\mathrm{ct}}^{\mathrm{M}}$ and $k_{\mathrm{ct}}^{\mathrm{W}}$ are the rate coefficients for chain transfer to monomer and water, respectively. The total rate of chain transfer $\left(R_{\mathrm{ct}}\right)$ can be expressed as follows:

$$
R_{\mathrm{ct}}=\left(k_{\mathrm{ct}}^{\mathrm{M}} \cdot(\mathrm{M}-\mathrm{SCOA})^{\mathrm{Gran}}+k_{\mathrm{ct}}^{\mathrm{W}} \cdot\left(\mathrm{H}_{2} \mathrm{O}\right)\right) \cdot\left(n^{*} \cdot N_{\mathrm{gran}} / N_{\mathrm{Av}}\right)
$$

For its hydrophilic nature, it is very likely that when a polymerase enzyme molecule has undergone chain transfer it desorbs into the cytoplasm. In the case of chain transfer to water, the resulting species is the free polymerase enzyme molecule (E-SH) which has to be initiated before it can enter the polymerization process again. The product of the chain transfer to monomer reaction is already initiated (E-S-M) and can directly undergo propagation in the cytoplasm.

\section{Molecular weight of polymer formed}

Since the polymerization of PHB is a chain reaction, the kinetic chain length $(v)$ of the PHB formed is given by Eq. (15):

$$
v=R_{\mathrm{p}} / R_{\mathrm{i}}
$$

Here we make the assumption that the system obeys steady-state conditions in the granule growth stage, i.e. the polymerase enzyme concentration at the granule surface $\left(n^{*}\right)$ does not change with time, since the rate of polymerization appears to be constant as will be shown in the next section.

$$
\mathrm{d}\left(n^{*} \cdot N_{\mathrm{gran}} / N_{\mathrm{Av}}\right) / \mathrm{d} t=0 \quad \text { and } \quad R_{\mathrm{ct}}=R_{\mathrm{i}}
$$

There are two processes which influence $n^{*}$, i. e. the adsorption of oligomeric polymerase enzyme from the cytoplasm onto the granule surface and chain transfer to 
water and/or monomer of a growing polymerase enzyme molecule at the granule surface and subsequent desorption of the reaction product. This results in the next equation:

$$
\mathrm{d}\left(n^{*} \cdot N_{\mathrm{gran}} / N_{\mathrm{Av}}\right) / \mathrm{d} t=R_{\mathrm{a}}-R_{\mathrm{ct}}
$$

From Eqs. (16) and (17) it follows that the rate of oligomeric polymerase enzyme adsorption onto granules $\left(R_{\mathrm{a}}\right)$ is equal to the rate of chain transfer of growing polymerase enzyme molecules at the granule surface $\left(R_{\mathrm{ct}}\right)$, when the system obeys steady-state conditions. Since the only chain stopping process is by chain transfer, the kinetic chain length is equal to the number-average degree of polymerization. The number-average molecular weight of the polymer formed can be visualized by Eq. (18):

$$
\begin{aligned}
\bar{M}_{\mathrm{n}} & =R_{\mathrm{p}} \cdot M_{0} / R_{\mathrm{ct}}=R_{\mathrm{p}} \cdot M_{0} / R_{\mathrm{a}} \\
& =\left(k_{\mathrm{p}} \cdot(\mathrm{M}-\mathrm{SCOA})^{\mathrm{Gran}} \cdot M_{0} \cdot n^{*}\right) /\left(k_{\mathrm{ads}} \cdot\left(\mathrm{E}-\mathrm{S}-\mathrm{M}_{z}\right) \cdot N_{\mathrm{Av}} \cdot \pi \cdot d_{\mathrm{gran}}^{2}\right)
\end{aligned}
$$

Here $M_{0}$ is the molecular weight of the repeating unit. According to Eq. (18) the number-average molecular weight of the polymer formed during the granule growth stage of the accumulation process depends solely on the granule diameter, since all other factors in Eq. (18) are constant.

\section{Granule growth: comparison with experiment}

\section{Rate of polymerization}

It is well known that Alcaligenes eutrophus can accumulate PHB up to about $80 \%$ of its cell dry weight. At this level any further accumulation proceeds at a negligible rate. It can be speculated that this is related to a diffusion limitation, caused by an increasing concentration of water soluble polymers present (e.g., DNA and RNA): at a polymer content of $80 \%$, the space available in the cell has been reduced to such an extent that further polymer accumulation would mean both massive polymer granule coalescence, and exclusion of other material from the bacterium, e.g. vital cell components such as DNA and proteins. This appears to limit the maximum polymer content achievable by the cell: how this limitation is achieved is as yet unknown.

The rate of polymerization has been calculated by considering the limitation discussed above. For modelling purposes, the conversion $X$ at the maximum polymer content achievable $\left(C_{\mathrm{PHB}}^{\mathrm{Max}}\right)$ will be defined as being 1 . So $X(t)=\left(C_{\mathrm{PHB}}^{\mathrm{t}}\right) /\left(C_{\mathrm{PHB}}^{\mathrm{Max}}\right)$ represents the fraction of polymer accumulated $\left(C_{\mathrm{PHB}}^{\mathrm{t}}\right)$ compared to the ultimate level achievable $\left(C_{\mathrm{PHB}}^{\mathrm{Max}}\right)$. Utilising this definition of conversion, the data of Ballard et al. ${ }^{6}$ and Kawaguchi and Doi ${ }^{28)}$ are converted into conversion-time curves (displayed in Fig. 3). From Fig. 3 the rates of polymerization have been calculated and are reported in Tab. 1. It can be seen in Fig. 3 and Tab. 1 that there are two separate regions; in both a constant rate of polymerization is observed.

The rates of polymerization in the first part for the fermentations reported by Ballard et al. ${ }^{6}$ and Kawaguchi and $\mathrm{Doi}^{28)}$ are almost the same. After a certain period the rate 


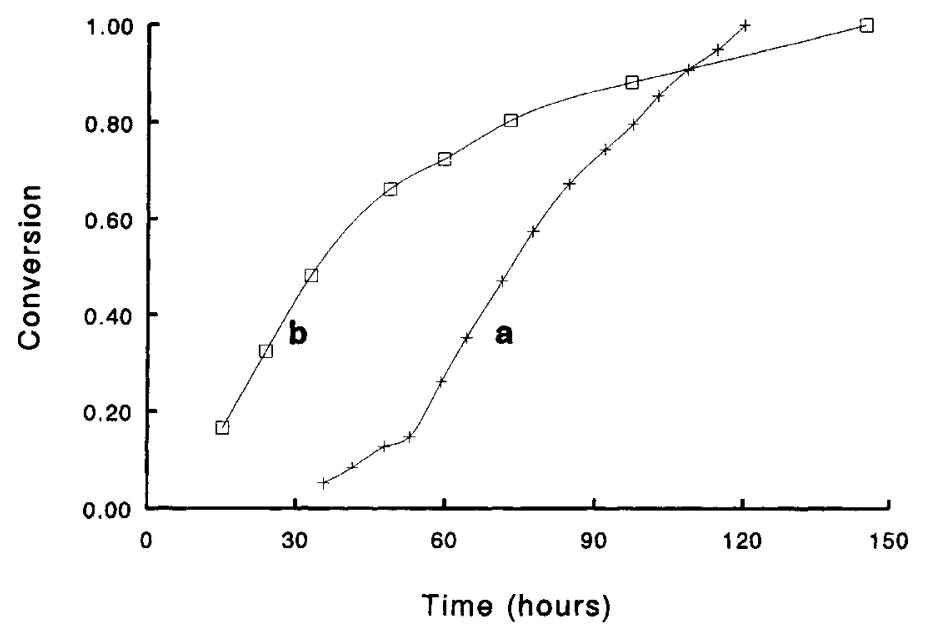

Fig. 3. Conversion-time curves for different PHB accumulations. $(+)$ Ballard et al. ${ }^{6)}$ (curve a) and ( $\square$ ) Kawaguchi and $D^{2}{ }^{28)}$ (curve b). Conversion is defined as the ratio of the fraction of polymer accumulated at time $t$ to the ultimate level of polymer achievable in the bacterium

Tab. 1. Rates of polymerization for the different fermentations in Fig. 3

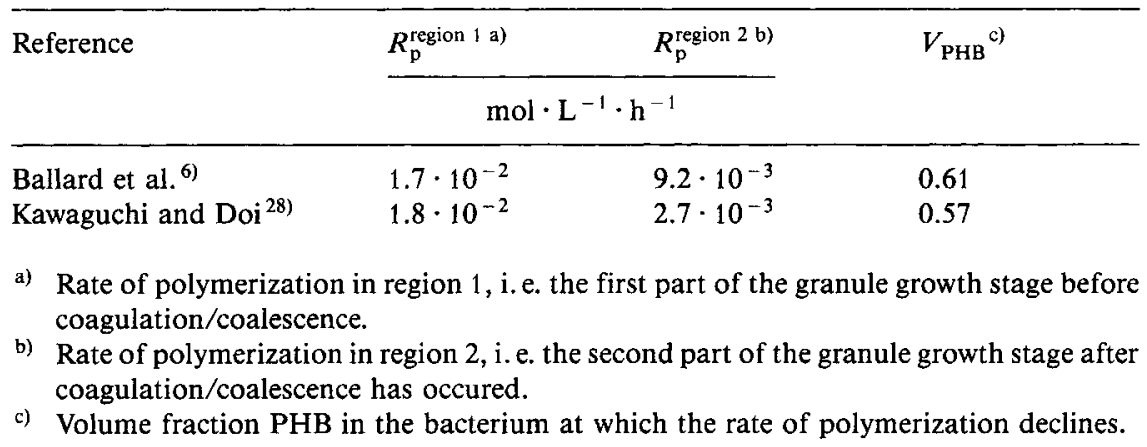

of polymerization gradually decreases to a second steady state, which continues until the end of the accumulation process.

One explanation for the two observed rates of polymerization is that - at a certain volume fraction of granules in the bacterium - close packing of granules occurs (see Fig. 4). Therefore, only coalescence would allow the accumulation of more polymer, since for monodisperse spheres hexagonal close packing occurs at a volume fraction spheres of 0.72 , whereas for polydisperse spheres fractions of dispersed phase can be above 0.9 . If, indeed, coalescence would occur during polymer accumulation then, according to Eq. (11), the rate of polymerization would decrease because the number 
Fig. 4. The degree of accumulation of $\mathrm{PHB}$ accumulating bacteria

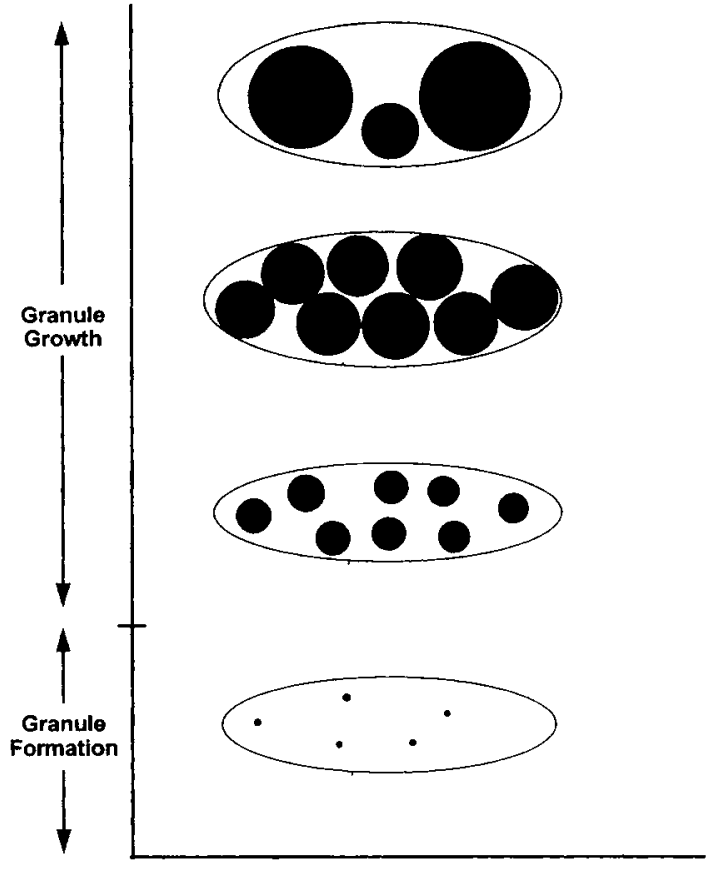

Degree of Accumulation

of granules $\left(N_{\text {gran }}\right)$ is reduced. This is exactly what is observed experimentally. The volume fraction of polymer in the bacterium equals 0.59 (average value, see Tab. 1) at the point where the rate of polymerization slows down. This is in good agreement with Ballard et al. ${ }^{6}$ who reported a value of 0.58 at which the bacteria's shape became more spherical, suggesting the onset of coalescence. This latter supposition, that the change in shape of the bacterium is related to coalescence, rests on the assumption that a bacterium changes shape because it is 'full' of granules. Further, the fact that the polymer is in an amorphous state ${ }^{33)}$ in the granules allows them to coagulate much easier than when the polymer would be in the crystalline state.

Ideally the concept of 'incipient coagulation' could be tested with a knowledge of the granule diameter as a function of conversion. Unfortunately these data do not exist, therefore rate and molecular weight data must be used to calculate the granule diameter $\left(d_{\text {gran }}\right)$ as a function of conversion. These model based 'experimental' data can be compared with simulated curves where no coagulation and controlled coagulation occur, respectively. This is done below.

a. Experimental: Granule diameter according to the volume growth rate. In the next approach we introduce mechanistic effects by using the volume growth rate coefficient $K^{34)}$. This coefficient may be evaluated from the knowledge of the propagation reaction and the conversion-time curve of the polymerization system. The mass of polymer added per unit of time to a single growing chain is given by 


$$
K=\left(k_{\mathrm{p}} \cdot(\mathrm{M}-\mathrm{SCoA})^{\mathrm{gran}} \cdot M_{0}\right) /\left(N_{\mathrm{Av}} \cdot \rho_{\mathrm{p}}\right)
$$

where $\rho_{\mathrm{p}}$ is the density of the polymer.

The volume growth rate coefficient will change during the polymerization, as can be seen in the extended form in Eq. (20) where the change in volume growth per unit of time is calculated once the molar conversion is known.

$$
\Delta K=K \cdot\left(X_{2}-X_{1}\right) \cdot\left(t_{2}-t_{1}\right)
$$

With Eq. (20) the increase in granule diameter per unit of time and conversion can be calculated according to

$$
\Delta d_{\mathrm{gran}}=(6 \cdot \Delta K / \pi)^{1 / 3}
$$

Once the granule size $d_{\text {gran, } 1}$ at time $t_{1}$ is known then the theoretical granule size $\left(d_{\text {gran, } 2}\right)$ at time $t_{2}$ and $X_{2}$ can be calculated

$$
d_{\mathrm{gran}, 2}=d_{\mathrm{gran}, 1}+\Delta d_{\mathrm{gran}}
$$

This value can subsequently be compared with the experimental value. Eqs. (19) - (22) are applied to conversion-time curves a and c from Fig. 3 and are represented by curve a in Figs. 5 and 6, respectively. Curve a in Figs. 5 and 6 shows a constant increase with conversion until a conversion of approximately 0.70 is reached after which the diameter increases more steeply.

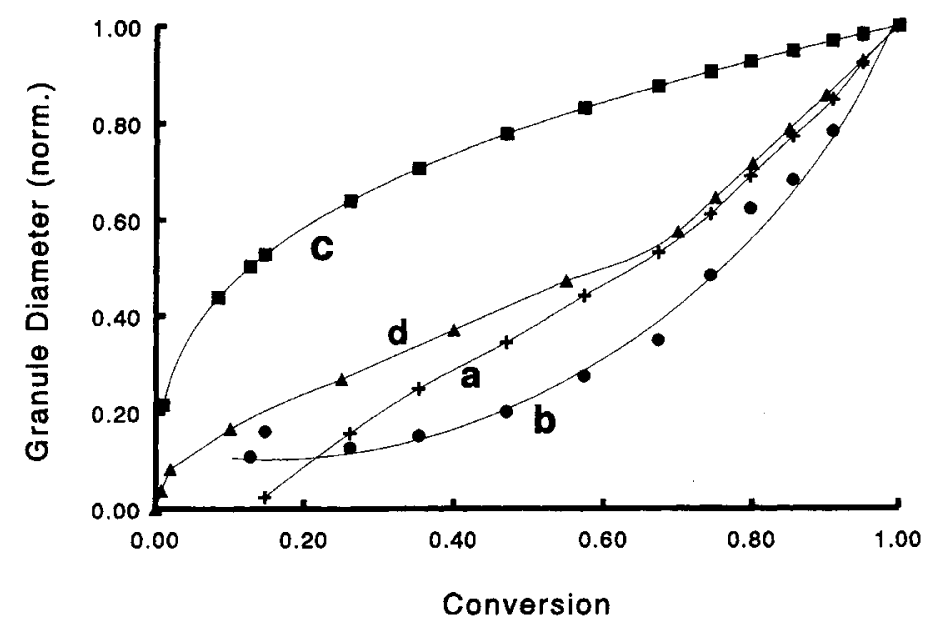

Fig. 5. The normalized granule diameter as a function of conversion. (+) Granule diameter according to Eqs. (19)-(22) (curve a), (-) granule diameter from the instantaneous number-average molecular weight data according to Eq. (23) (curve b), (E) calculated granule diameter according to Eq. (24) (curve c), ( $\mathbf{\Delta}$ ) theoretical latex particle diameter if coagulation is simulated for a conventional emulsion polymerization, number of latex particles decreased by a factor of 5 after $70 \%$ conversion (curve d). Experimental input data from Ballard et al. ${ }^{6}$ ) 


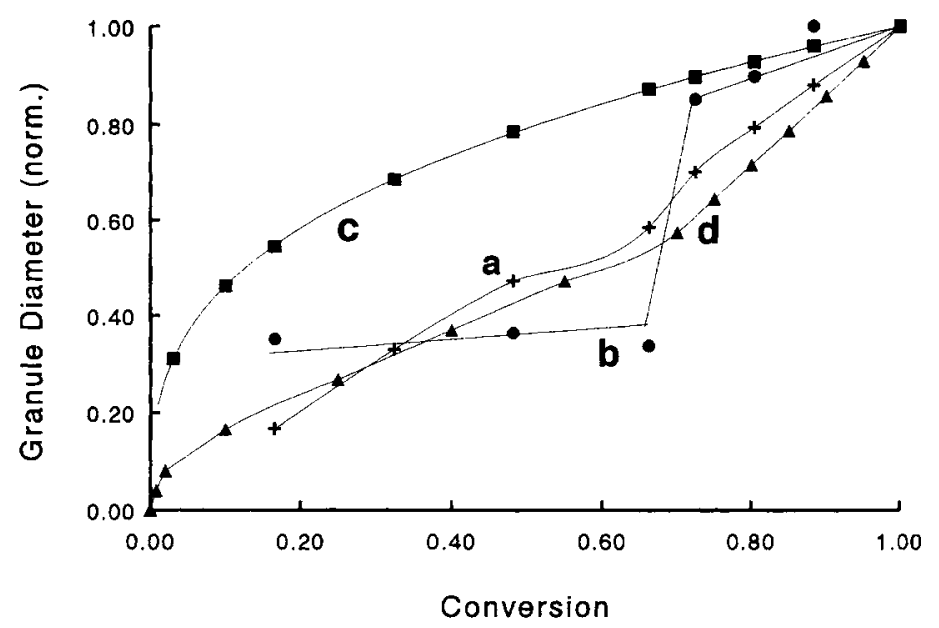

Fig. 6. The normalized granule diameter as a function of conversion. (+) Granule diameter according to Eqs. (19)-(22) (curve a), (•) granule diameter from the instantaneous number-average molecular weight data according to Eq. (23) (curve b), (ם) calculated granule diameter according to Eq. (24) (curve c), (A) theoretical latex particle diameter if coagulation is simulated for a conventional emulsion polymerization, number of latex particles decreased by a factor of 5 after $70 \%$ conversion (curve d). Experimental input data from Kawaguchi and $\mathrm{Doi}^{28)}$

b. Experimental: Granule diameter calculated from the molecular weight. The last method by which the diameter of the granules can be determined is by using the experimental molecular weight data. According to Eq. (18) the granule diameter is a function of the number-average molecular weight $\left(\bar{M}_{\mathrm{n}}\right)$ of the polymer formed, rewriting Eq. (18) gives Eq. (23):

$$
d_{\mathrm{gran}}=\sqrt{B / \bar{M}_{\mathrm{n}}}
$$

Here the constant $B$ is comprised of different parameters that are assumed to be constant during the accumulation process, such as $M_{0},(\mathrm{M}-\mathrm{SCoA})^{\mathrm{Gran}}, n^{*}, k_{\mathrm{p}}, \mathrm{k}_{\text {ads }}$ and $\left(\mathrm{E}-\mathrm{S}-\mathrm{M}_{z}\right)$. Eq. (23) is applied to the instantaneous number-average molecular weights given in Figs. 7 and 8 which are represented in Figs. 5 and 6 by curve b, respectively. The data in Figs. 5 and 6 are normalized to the last data point, concomitantly it is possible to plot the data without detailed knowledge of the value of the constant $B$. Curve $\mathrm{b}$ in Fig. 5 shows a constant increase with conversion until a conversion of approximately 0.70 is reached after which the diameter increases more steeply. In the case of curve b in Fig. 6 this behaviour, i.e. an increase in diameter up to a conversion of approximately 0.70 and then a more steeper increase, is less pronounced. This is probably due to the fact that less experimental data were available in the first part of the accumulation process, i.e. the part before coagulation occurs. 
c. Simulation: Course of granule diameter as a function of conversion. It can be shown that if monomer is converted into polymer and this polymer is then put into a sphere, i. e. a granule, the resulting granule diameter is given by Eq. (24):

$$
d_{\text {gran }}=\left(\left(6 \cdot M_{0} \cdot n_{\mathrm{m}}^{X=1} \cdot X\right) /\left(\pi \cdot \rho_{\mathrm{p}}\right)\right)^{1 / 3}
$$

$n_{\mathrm{m}}^{X=1}$ is the total number of moles of monomer converted into polymer at a conversion equal to 1. Eq. (24) is represented by curve $\mathrm{c}$ in Figs. 5 and 6 . The diameter of the growing granule increases strongly in the beginning and gradually reaches its maximum value at the end of the polymerization.

d. Simulation: Latex particle diameter during an emulsion polymerization with coagulation. Curve $d$ in Figs. 5 and 6 represents the latex particle diameter of a simulated emulsion polymerization where after $70 \%$ conversion coagulation is simulated. This coagulation decreased the number of latex particles per litre by a factor of 5 .

From the above it can be seen that both 'experimental' curves follow more closely the simulation where coagulation occurred. We believe this is evidence for both the assumption that coagulation occurs during polymer accumulation, and also for the kinetic model derived in this paper. Further testing of these ideas will necessarily involve the measurement of granule size as a function of conversion.

\section{Molecular weight}

According to Eq. (18) the molecular weight of the PHB formed during the granule growth stage has to decrease as the granules grow, i. e. with increasing granule diameter. In Figs. 7 and 8 the cumulative and instantaneous number-average molecular weights

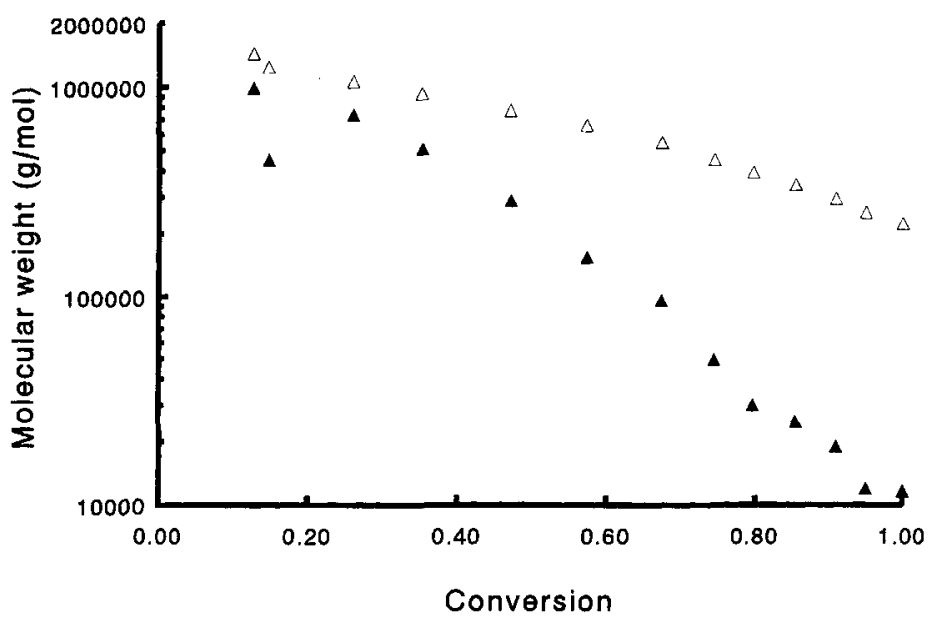

Fig. 7. Instantaneous and cumulative number-average molecular weight of PHB formed as a function of conversion. Ballard et al. ${ }^{6)}:(\triangle)$ cumulative number-average molecular weight, $(\mathbf{\Delta})$ instantaneous number-average molecular weight 


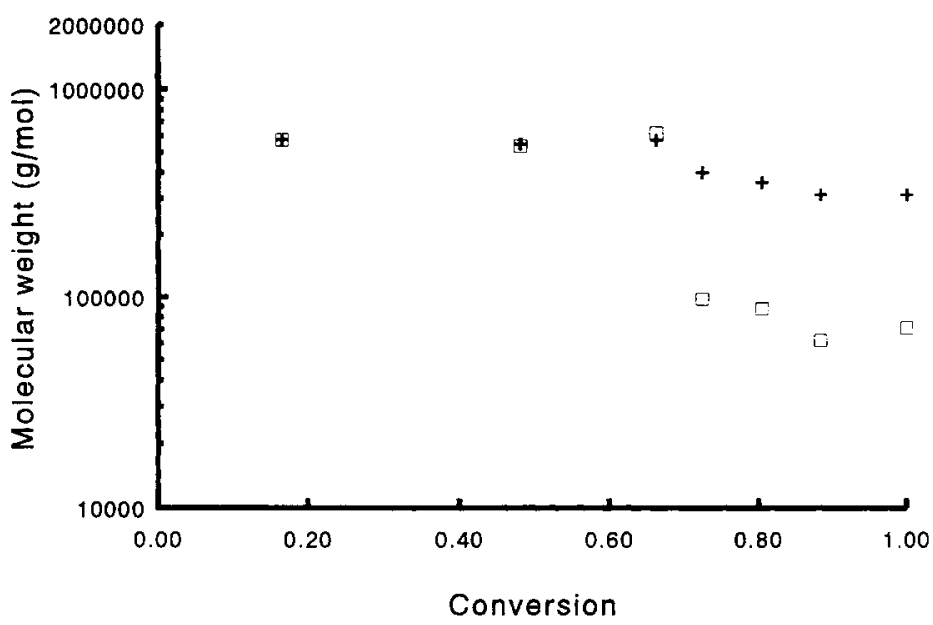

Fig. 8. Instantaneous and cumulative number-average molecular weight of PHB formed as a function of conversion. Kawaguchi and $\mathrm{Doi}^{28)}:(+)$ cumulative number-average molecular weight, $(\square)$ instantaneous number-average molecular weight

of PHB formed during two different fermentations as a function of conversion are plotted $^{6,28)}$. Figs. 7 and 8 show that the molecular weight of the PHB formed decreases with conversion, as predicted by the model. The fact the the molecular weight shows a stronger decrease after approximately $70 \%$ conversion can be explained by the fact that coagulation occurs, as already pointed out in the previous section. When this coagulation occurs, the number of granules decreases while the diameter of the remaining granules increases. This results initially in a sudden decrease of the molecular weight which will decrease constantly as the rate of polymerization becomes constant again, i.e. $N_{\text {gran }}$ is constant again. After the coagulation the decrease of the molecular weight is solely determined by the increase of the granule diameter due to the formation of new polymer.

\section{Conclusions}

In the foregoing sections we developed a new kinetic model which is able to describe the experimental data on PHB accumulation in Alcaligenes eutrophus reported in open literature. In contrast to previous models, the heterogeneous nature of the PHB accumulation is taken into account. The accumulation of PHB is projected on different aspects known to be important in the conventional emulsion polymerization process, such as particle formation and interval II kinetics. Further, adsorption and desorption of growing and terminated polymerase enzyme species are believed to play an important role in the PHB accumulation process. The experimentally observed decrease in the rate of polymerization can be explained by coalescence of the granules present due 
to the fact that these granules reach a pseudo-close packing in the accumulating bacterium. Evidence is given for the occurrence of coalescence of these granules during the accumulation process by two independent approaches, i. e. via the growth factor $K$ and the relation between the molecular weight of the polymer formed and the granule diameter.

The decrease in the molecular weight of the polymer formed during the PHB accumulation process can fully be explained in terms of the kinetics of the polymerization process and the effect of the granule size thereon. This is in contrast with the explanation that the decrease in molecular weight is due to the simultaneous operation of the polymerase enzyme and the depolymerase enzyme ${ }^{35,36)}$

The developed model focuses on the PHB accumulating bacterium Alcaligenes eutrophus, but we believe that this model might also be applicable to other PHB accumulating species.

The authors would like to thank Dr. Gerard van Doremaele, Roy Janssen, Dr. Annemieke Aerdts, Bart van Bilsen and John Geurts for their inspiring discussions. Special thanks goes to Prof. Piet J. Lemstra who gave the "onset" to this investigation.

1) M. Lemoigne, Ann. Inst. Pasteur Paris 39, 144 (1925)

2) E. A. Dawes, P. J. Senior, Adv. Microbiol. Physiol. 10, 135 (1973)

3) A. J. Anderson, E. A. Dawes, Microbiol. Rev. 54, 450 (1990)

4) US 1,732,795 (1927), Goodyear Tire and Rubber Co., inv.: R. P. Dinsmore; Chem. Abstr. 24, 266 (1927)

5) D. Ellar, D. G. Lundgren, K. Okamura, R. H. Marchessault, J. Mol. Biol. 35, 489 (1968)

6) D. G. H. Ballard, P. A. Holmes, P. J. Senior, NATO ASI Ser., Ser. C 215, 293 (1987)

7) T. U. Gerngross, P. Reilly, J. Stubbe, A. J. Sinskey, O. P. Peoples, J. Bacteriol. 175, 5289 (1993)

8) G. J. M. De Koning, I. A. Maxwell, J. Environ. Polym. Degrad. 3, 223 (1993)

9) J. Kurja, H. F. Zirkzee, I. A. Maxwell, in preparation

10) D. R. Weyenberg, D. E. Findlay, J. Cekada, A. E. Bey, J. Polym. Sci., Polym. Symp. 27, 27 (1969)

11) US 4,273,634 (1981), Dow Corning Corp., invs.: J. C. Saam, R. L. Wegener; Chem. Abstr. 95, 82044r (1981)

12) J. C. Saam, D. J. Huebner, J. Polym. Sci., Polym. Chem. Ed. 20, 3351 (1982)

13) S.-Y. Lu, P. Quayle, C. Booth, S. G. Yeates, J. C. Padget, Polym. Int. 32, 1 (1993)

14) I. A. Maxwell, B. R. Morrison, R. G. Gilbert, D. H. Napper, Macromolecules 24, 1629 (1991)

15) F. K. Hansen, J. Ugelstad, "Particle Formation Mechanisms"; in "Emulsion Polymerization"; I. Piirma Ed., Academic Press, New York 1982

16) R. G. Gilbert, D. H. Napper, J. Macromol. Sci. - Rev. Macromol. Chem. Phys. C23, 127 (1983)

17) D. C. Blackley, “Emulsion Polymerization”; Applied Science Publishers LTD, London 1975

18) W. V. Smith, R. H. Ewart, J. Chem. Phys. 16, 592 (1948)

19) W. D. Harkins, J. Am. Chem. Soc. 69, 1428 (1947) 
20) J. W. Goodwin, J. Hearn, C. C. Ho, R. M. Ottewill, Colloid Polym. Sci. 252, 464 (1974)

21) R. M. Fitch, C. H. Tsai, in "Polymer Colloids", R. M. Fitch Ed., Plenum, New York 1971

22) J. Ugelstad, F. K. Hansen, Rubber Chem. Technol. 49, 536 (1976)

23) S. J. McCarthy, E. E. Elbing, I. R. Wilson, R. G. Gilbert, D. H. Napper, D. F. Sanger, Macromolecules 19, 2440 (1986)

24) J. H. T. Luong, A. Mulchandani, A. Leduy, Enzyme Microb. Technol. 10, 326 (1988)

25) A. Mulchandani, J. H. T. Luong, C. Groom, Appl. Microbiol. Biotechnol. 30, 11 (1989)

26) A. V. Quinlan, Ann. N. Y. Acad. Sci. 469, 259 (1986)

27) D. F. Ollis, Ann. N. Y. Acad. Sci. 413, 144 (1983)

28) Y. Kawaguchi, Y. Doi, Macromolecules 25, 2324 (1992)

29) R. Griebel, Z. Smith, J. M. Merrick, Biochemistry 7, 3676 (1968)

${ }^{30)}$ G. W. Haywood, A. J. Anderson, E. A. Dawes, FEMS Microbiol. Lett. 57, 1 (1989)

31) P. J. Flory, "Principles of Polymer Chemistry", Cornell University Press, New York 1953

32) A. Fiechter, TIBTECH 10, 208 (1992)

33) G. J. M. De Koning, P. J. Lemstra, Polymer 33, 3292 (1992)

34) G. Lichti, B. S. Hawkett, R. G. Gilbert, D. H. Napper, J. Polym. Sci., Polym. Chem. Ed. 19,925 (1981)

35) Y. Doi, Y. Kawaguchi, M. Kunioka, FEMS Microbiol. Lett. 67, 165 (1990)

36) Y. Doi, "Microbial Polyesters", VCH Publishers, Inc., New York 1990 